\title{
Ventricular pre-excitation in the general population: a study on the mode of presentation and clinical course
}

\author{
J A Goudevenos, C S Katsouras, G Graekas, O Argiri, V Giogiakas, D A Sideris
}

Objective-To describe the mode of presentation and the clinical course of patients with ventricular pre-excitation (Wolff-Parkinson-White (WPW) syndrome), with special emphasis on asymptomatic cases in the general population.

Methods-Over an eight year period (1990-97) a prospective population based survey of cases with WPW pattern was conducted in a defined population in north west Greece (340 000 inhabitants). ECGs with WPW pattern were obtained from a widespread pool of ECGs within the health system. Results-During the study period, 157 cases with WPW pattern were identified (49 female, 108 male). Ages ranged from infants to 84 years, mean (SD) 49.1 (21.0) years in female and 39.6 (20.6) years in male subjects $(\mathrm{p}<0.01) ; 78(49 \%)$ had no history of syndrome related symptoms. Asymptomatic subjects ( $\mathrm{n}=77 ; 24$ female, 53 male) were older than symptomatic subjects (mean age $46.7(21.0) \cup 38.5$ (20.6) years, $\mathrm{p}<0.03)$. Documented supraventricular tachycardia was recorded in 27 patients $(17 \%)$ and atrial fibrillation in $12(8 \%)$ (mean age at first episode 31.2 (18.3) and 51.6 (20.7) years, respectively, p < 0.01). During follow up (mean 55 months) no case of sudden death occurred. Three asymptomatic subjects reported episodes of brief palpitation.

Conclusions-WPW pattern is more common, and diagnosed at a younger age, in men than in women. About half the patients with WPW pattern on ECG are asymptomatic at diagnosis and tend to remain so thereafter. No sudden cardiac death occurred during the study period.

(Heart 2000;83:29-34)

Keywords: Wolff-Parkinson-White syndrome; epidemiology

Pre-excitation is caused by an anomalous atrioventricular connection capable of antegrade and usually retrograde conduction. A delta wave is the main electrocardiographic abnormality, together with a short PR interval. Wolff-Parkinson-White (WPW) syndrome involves patients with both pre-excitation in the surface ECG and symptoms caused by arrhythmias, either regular (circus movement tachycardias) or irregular (atrial fibrillation). The incidence of WPW pattern varies from 0.1 to 3 per 1000 ECGs. $^{12}$ The incidence of paroxysmal tachycardias in the young adult population is approximately $10 \%$ and increases with age up to $30 \% .^{3}$ Episodes of atrial fibrillation occur in as many as $20-30 \%$ of patients with the syndrome. ${ }^{4}$ The exact risk for developing ventricular fibrillation during high ventricular rates is not known. Few studies have been done on patients with pre-excitation to establish its natural history. ${ }^{5-7}$ However, knowledge about the mode of presentation and clinical course of patients with ventricular preexcitation remains limited, especially in asymptomatic subjects. Most of the available data come from tertiary centre studies biased by referrals of symptomatic patients with the WPW syndrome - that is, both ECG evidence of ventricular pre-excitation and a history of tachycardia.

Our aim in this study was to describe the mode of presentation and the clinical course of persons with the WPW pattern, with particular emphasis on asymptomatic individuals in the general population of a geographical region of Greece.

\section{Methods}

The study was conducted in a confined geographical region in north west Greece, with an area of $8500 \mathrm{~km}^{2}$ and a known population of 340000 inhabitants, stratified for age and sex (51\% female, $49 \%$ male; 1991 Greek census). Population based epidemiological research is feasible in this area for a variety of reasons: the population is fairly stable with no migratory fluxes and the combination of natural geographical boundaries and the lack of proximity to the referral medical centres allows medical evaluation of the majority of patients within the region. Health care is provided by private clinics, 20 small scale primary care health centres, four public hospitals, and a single tertiary care hospital (University of Ioannina Hospital). For the study period no electrophysiology studies or ablations were carried out in this region in patients with pre-excitation.

From January 1990 to April 1997, the majority of routine ECGs obtained within our public health system were studied and all cases where there was ECG evidence of ventricular pre-excitation were recorded. ECGs were studied in an unselected population within the health system. As an ECG is part of health examination of many individuals seeking employment, athletes participating in competitive sports, and students entering university, an ECG is recorded in a large population of asymptomatic individuals. ECGs were also obtained from patients presented in physicians' offices, clinics, health centres, or preoperatively, with symptoms not necessarily related to cardiovascular disease. Most of ECGs taken in 
this population were screened, as all general practitioners as well as specialists and residents in cardiology were informed of and kept reminded about the study. All patients undergoing an operation had an ECG taken preoperatively. In this subpopulation we examined the prevalence of pre-excitation and used this as an internal control for the study. We estimated the incidence of new cases with a WPW pattern on ECG revealed preoperatively, because this group was clearly not screened for the syndrome, and for asymptomatic individuals with a WPW pattern. All ECGs were analysed by cardiologists. The criteria for the diagnosis of pre-excitation, based on the 12 lead surface ECG, were a shortened PR interval (less than $120 \mathrm{~ms}$ ) and a typical slurred upstroke of the initial QRS complex (delta wave) with a consequent widening of the QRS complex (QRS duration > $100 \mathrm{~ms}$ ). The ECGs with a WPW pattern were reviewed and coded independently by two cardiologists. Accessory pathway location was established by the use of ECG criteria according to Fitzpatrick's algorithm. ${ }^{8}$ The start of the follow up period dated from the time when the first ECG with a delta wave was recorded.

All patients enrolled in the study underwent a full medical evaluation. They were asked particularly about symptoms that could be related to episodes of tachycardia (including palpitations or syncope), cardioactive drugs, and previous attempts at surgical or transcatheter ablation. All patients underwent a careful physical examination and echocardiographic evaluation to screen for the presence of structural heart disease. Participants in the study were subsequently followed by phone or through outpatient clinic visits at six month intervals. Any change in clinical status (for example, the onset of new symptoms, ablation procedures, and so on) was included in the study record. Data collection was completed through 1998; follow up was achieved in 96\% of the group.

\section{STATISTICS}

The incidence of new cases with a WPW pattern was calculated by dividing the number of new recognised WPW cases by the estimated population in the subgroup according to the equation: incidence $=$ (number of new cases occurring in period/number of persons at risk $\times$ length of period) $\times 10^{\mathrm{n}}$. ${ }^{9}$

Continuous variables are expressed as mean (SD) and analysed by the Student $t$ test. Nominal findings were evaluated by the $\chi^{2}$ test with Yates correction when needed. A p value of $<0.05$ was considered significant.

\section{Results}

The study population consisted of 157 individuals, with a significant male predominance (108 male $(68.8 \%)$ v 49 female $(31.2 \%)$ $(p<0.001))$. The age at diagnosis ranged from infancy to 84 years. The mean (SD) age at recruitment was 42.5 (21.1) years: $39.6(20.6)$ years in male subjects and 49.1 (1.0) years in female subjects; $\mathrm{p}<0.01$ (table 1 ).
Table 1 Age at diagnosis and sex of residents of north west Greece with Wolff-Parkinson-White pattern

\begin{tabular}{llll}
\hline & Male & Female & p Value \\
\hline $\mathrm{n}(\%)$ & $108(69)$ & $49(31)$ & $<0.001$ \\
Age at diagnosis (years) & to 84 & 6 to 83 & - \\
$\begin{array}{l}\text { Range } \\
\text { Mean (SD) }\end{array}$ & $39.6(20.6)$ & $49.1(21.0)$ & $<0.01$ \\
\hline
\end{tabular}

Of the 157 individuals recruited, 121 (82\%) were first diagnosed at study entry. The incidence for new cases was 4.4/100 000/year. The incidence in male subjects $(6.8 / 100000 /$ year) was more than twice that in female subjects (2.2/100 000/year).

The age distribution (fig 1 ) for male subjects did not differ significantly from a theoretically even age distribution, but a greater detection rate in female subjects was seen during the seventh decade of life $(14 / 41, \mathrm{p}<0.05)$. Total follow up time was 720 patient-years (approximately 55 months/patient).

SYMPTOMS

Eighty patients ( $51 \%$; 55 male and 25 female) were symptomatic at diagnosis (77) or developed symptoms (brief palpitations) during the follow up period (3). Symptoms were palpitations in 65, history of syncope in 10 , and both in five. Seventy seven patients $(49 \%)$ were totally asymptomatic (53 male and 24 female). Mean (SD) age at study entry was 38.5 (20.6) years for symptomatic subjects $v 46.7$ (21.0) for the asymptomatic subjects $(\mathrm{p}<0.03)$.

\section{ARRHYTHMIAS}

Thirty nine arrhythmias were documented in 38 patients $(24.2 \%), 31$ male (mean age 34.1 (19.2) years) and seven female (mean age 52.3 (22.0) years). The difference between the two sexes was not significant either in incidence or in age. Supraventricular tachycardias (orthodromic and antidromic) were recorded in 27 patients (mean age 31.5 (19.4) years), including two infants, 23 men (mean age $31.2(17.4$ ) years), and four women (mean age 36.3 (13.0) years). Antidromic supraventricular tachycardias were documented in two patients. Atrial fibrillation was recorded in 12 patients (mean age 52.1 (20.5) years), nine men (mean age 46.9 (19.8) years) and three women (mean age 68.0 (13.9) years) (fig 2). In 11 of 12 cases, atrial fibrillation was the first arrhythmic event and in one man with a history of supraventricular tachycardias, atrial fibrillation appeared

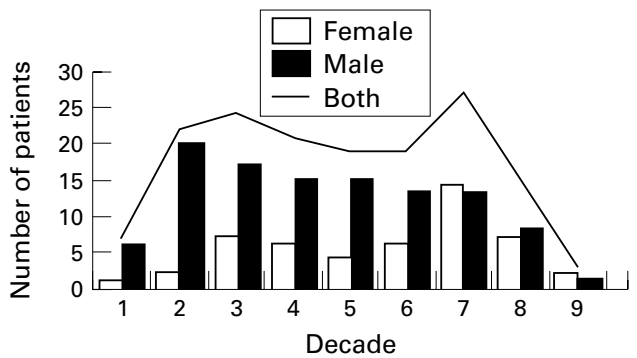

Figure 1 Age distribution at diagnosis of Wolff-Parkinson-White pattern among residents of north west Greece. 


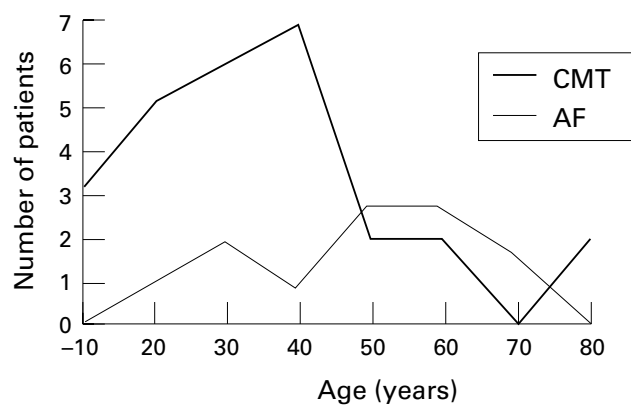

Figure 2 Age at onset of first arrhythmic event in patients with Wolff-Parkinson-White syndrome in north west Greece. AF, atrial fibrillation; CMT, circus movement tachycardia.

later. Onset of atrial fibrillation appeared in older patients than supraventricular tachycardia $(p<0.01$, table 2$)$. Three patients with atrial fibrillation (1) or supraventricular tachycardia (2) also had a history of syncope or near syncope.

\section{ACCESSORY PATHWAY LOCATION}

There was an excess of left sided (54\%) $v$ right sided $(46 \%)$ pathways: left anterolateral, 35 (22\%); left posterolateral, 27 (17\%); left posteroseptal, 20 (13\%); right anterolateral, 20 (13\%); right midseptal, 21 (13\%); right posteroseptal, 13 (8\%); right anteroseptal, 12 $(8 \%)$. Forty two per cent of the bypass tracts were septally located. We were not able to identify the accessory pathway location in nine ECGs $(6 \%)$. There was no difference in age or sex among the eight groups of pathway location.

The accessory pathway location in patients with supraventricular tachycardias was: left lateral, 14; septal, 12; right lateral, 3; in patients with atrial fibrillation it was: septal, 6; left lateral, 5; right lateral, 1 . Ten $(67 \%)$ of the patients with syncope or near syncope had a septally located accessory pathway.

COEXISTENCE OF CARDIAC DISEASE

Thirteen patients had a history of cardiac disease: nine had coronary artery disease, two had mitral regurgitation, one had mitral valve prolapse, and one had cardiomyopathy associated with supraventricular tachycardia. None of the patients had congenital heart disease.

PREGNANCY

We identified four pregnant women (three asymptomatic, one with palpitations) with WPW pattern. A fifth woman, with known symptomatic WPW syndrome, experienced more palpitations during pregnancy.

Table 2 Sex, age, and pathway location of patients with circus movement tachycardias or atrial fibrillation

\begin{tabular}{llll}
\hline & AF & CMT & $p$ Value \\
\hline $\mathrm{n}$ & 12 & 27 & \\
Sex (male) & $9(75 \%)$ & $23(85 \%)$ & NS \\
Age (years) (mean (SD)) & $52.1(20.5)$ & $31.5(19.4)$ & $<0.001$ \\
Pathway location (left/septal/right) & $5 / 6 / 1$ & $11 / 12 / 2$ & NS
\end{tabular}

$\mathrm{AF}$, atrial fibrillation; CMT, circus movement tachycardia.
WPW PATTERN IN CANDIDATES FOR OPERATIONS Preoperatively we identified 19 new cases of WPW pattern (14 men, five women). The mean age was 41.4 (22.4) years for men and 56.2 (15.1) years for women. There was no significant difference in age at diagnosis or sex between these patients and the remainder (age at diagnosis, 39.3 (21.1) years for male patients $(\mathrm{n}=94)$ and $49.7(21.3)$ years for female patients $(\mathrm{n}=44))$. Although the incidence of newly detected WPW in this group appeared somewhat increased (5.4/100 000 candidates/ year), it was not significantly different from the overall incidence in north west Greece.

MEDICAL TREATMENT

Sixteen patients ( 11 men and five women) were receiving medical treatment. Drugs used were amiodarone (7), sotalol (1), propafenone (3), disopyramide (1), verapamil (2), and $\beta$ blockers (2). Compliance with the drug regimen was relatively poor in this group.

CLINICAL COURSE

Based on a mean follow up period of 55 months (range 20 to 108 months), the cumulative observation period was 720 patient-years. During the study period three of the 80 asymptomatic patients $(3.7 \%)$ had palpitations. Ten patients (nine men, one woman), mean age 38.5 (17.1) years, nine of whom were symptomatic, underwent transcatheter ablation of the accessory connection using radiofrequency energy. The indication for the procedure was recurrent episodes of tachycardia in eight patients (three with atrial fibrillation, five with supraventricular tachycardias) and syncope in one patient. Ablation was also performed in one asymptomatic athlete involved in competitive sports. There were three recurrences of ventricular pre-excitation after the intervention.

Six patients died during follow up, none suddenly. Fatal myocardial infarction was responsible in two cases, malignancy in three, and intracranial haemorrhage in one. One of these six patients, who died during a colon cancer operation, had a history of palpitations.

\section{Discussion}

\section{DEMOGRAPHIC FEATURES}

This study involved a follow up of a group of patients with pre-excitation referred to the national health system services of north west Greece for various, not purely cardiac, reasons. The incidence for new cases was 4.4/100 000/ year and the incidence in males was more than twice that in females. However, it is nor possible to determine the precise incidence of WPW pattern because of its intermittent nature. The incidence found in our study was approximately the same as the overall incidence of new cases of WPW in the Olmsted County study. ${ }^{5}$ The increased frequency in males is in accordance with previous reports ${ }^{3510}$; however, Sano et al recently reported a 1:1 male to female ratio in Japanese schoolchildren with ventricular pre-excitation. ${ }^{11}$ The highest incidence in female subjects in our study was during the seventh decade (fig 1). Interestingly this peak 
has not been reported in other studies. Men were significantly younger than women at first diagnosis. The reasons for these sex differences are not known but it has been suggested that women are cared for and treated differently for a variety of diseases. In Greece, women live longer than men and there are no differences between them in health insurance. Despite the fact that unpublished data from our area have shown no sex bias for coronary intervention (Goudevenos J, Giogiakas V, Michalis LK, et al. XIX Congress of the European Society of Cardiology, Stockholm, August 24-28, 1997; Goudevenos J, Pappas K, Giogiakas V, et al. XXth Congress of the European Society of Cardiology, Vienna, August 22-26, 1998), we cannot exclude the possibility that women's access to health care is less or later in their life than men's. However, the fact that the overall incidence, male to female ratio, and mean age at diagnosis were not different from those in the preoperative cases (who were clearly not screened for the WPW syndrome) may indicate that our findings in the general population were fairly representative.

SUDDEN DEATH

Sudden death is fortunately a rare event but may be the first manifestation of the syndrome in previously asymptomatic individuals. ${ }^{12}$ The estimated incidence in both patients with pre-excitation pattern and those with complete WPW syndrome ranges from 0 to 0.006 per patient-year. ${ }^{3-7}{ }^{13}$ In the present study no sudden death occurred in 720 patient-years. None of the six deaths reported was attributable to a primary arrhythmia. There may be various reasons for the absence of this fatal complication. First, 720 patient-years is not enough. Second, the majority of patients were asymptomatic or nearly asymptomatic; previous studies have shown that patients who were asymptomatic at diagnosis do extremely well and are not liable to sudden cardiac death. ${ }^{5}{ }^{10}$ Third, most of the study participants had no organic disease. Finally, some individuals with a bypass tract would not have been identified, so patients with WPW syndrome may have died suddenly without an ECG diagnosis having been made. Topaz et al showed that among 23 young patients resuscitated from sudden cardiac death, three (14\%) had WPW syndrome, but it is worth noting that two of these three patients had a past history of palpitations. ${ }^{14}$

ATRIAL FIBRILLATION

Atrial fibrillation is not a rare arrhythmia in WPW syndrome. It is clinically important because extremely rapid rates can occur over the bypass tract, leading to haemodynamic deterioration or ventricular fibrillation. In the present study, 12 patients $(8.0 \%)$ had atrial fibrillation. The incidence of atrial fibrillation was somewhat lower than other values reported $(11 \%$ to $30 \%){ }^{15-19}$ mainly because of the relatively large number of asymptomatic patients in our study. The mean age of the atrial fibrillation group was greater than that of patients with circus movement tachycardias.
SUPRAVENTRICULAR TACHYCARDIAS

The most common tachycardias associated with WPW syndrome are circus movement tachycardias. ${ }^{20}$ In our study $93 \%$ of these were orthodromic and this is in accordance with previous studies.

SYMPTOMS

Almost half of our study cohort reported symptoms. The symptomatic patients were significantly younger than the asymptomatic subjects. Syncope occurred in 15 individuals $(9.5 \%)$. According to previous studies, ${ }^{21-23}$ syncope occurs in $11 \%$ to $29 \%$ of patients with WPW syndrome. Ten $(67 \%)$ of the patients with syncope or near syncope had a septally located accessory pathway. Timmermans et al reported that the dense innervation of this area could cause extreme shortening of the refractory period of the accessory pathway leading to very fast ventricular response during atrial fibrillation. ${ }^{12}$ We found no difference in sex or age between patients with and without syncope, and these findings are in agreement with other series. $^{24}$

COEXISTENCE OF CARDIAC DISEASE

The most common associated cardiac disease was coronary artery disease $(6 \%)$. We did not find any patient with WPW pattern and congenital heart disease. Although an association between congenital heart lesions with WPW pattern has been reported, ${ }^{525}$ our findings are supported by Sano et al, ${ }^{11}$ suggesting that the combination in a community based population is uncommon.

\section{PREGNANCY}

Pregnancy seems to increase the likelihood of arrhythmias and symptoms in women with WPW syndrome. ${ }^{26} 27$ This is because of changes in haemodynamics, hormonal function, and sympathetic tone (increased stress and anxiety). In our study, pregnancy induced symptoms in one previously asymptomatic woman and increased the frequency of palpitations in another.

\section{SYMPTOM STATUS AT DIAGNOSIS AND CLINICAL} COURSE

There was approximately a 1:1 ratio between asymptomatic and symptomatic patients, and the most common presenting symptom in the latter group was palpitations. In previous retrospective studies, symptoms have been noted in $60-64 \%$ of patients with pre-excitation identified through hospital records. ${ }^{78}$ In our study, three individuals asymptomatic at diagnosis developed symptoms (brief palpitations) during the follow up period. This finding suggests that patients asymptomatic at diagnosis tend to remain free of symptoms or tachycardias and have a generally benign prognosis. There is growing interest in the prognosis of asymptomatic subjects with WPW pattern, principally because potentially curative radio frequency ablation might be a management possibility. Several studies have attempted to assess the risk of symptom development in individuals who are asymptomatic at the time of diagnosis 
of ventricular pre-excitation. Munger et al reported that $30 \%$ of initially asymptomatic individuals developed symptoms related to arrhythmias over a 12 year follow up period. ${ }^{5}$ In concordance with our findings, other investigators have reported even lower rates of symptom development $(0-8 \%)$. However, follow up periods have been variable. ${ }^{29}{ }^{30}$ More importantly, investigators have attempted to assess the risk of sudden cardiac death in asymptomatic patients. In particular, some studies have reported that this fatal event is extremely rare among asymptomatic individuals with WPW pattern, but may occur. ${ }^{361431}$ Recently, Timmermans et al reported that 15 of 690 patients $(2.2 \%)$ with WPW syndrome who were referred to their hospital over a 16 year period had an aborted sudden cardiac death, and ventricular fibrillation was the first clinical manifestation in eight patients. ${ }^{12}$ However, that study was a retrospective one, the patients representing a selected population referred to hospital for evaluation and treatment of an arrhythmia, and information about the incidence of sudden cardiac death in asymptomatic individuals with known WPW pattern could not possibly have been obtained. It is not clear also whether the WPW patients who were resuscitated from ventricular fibrillation did or did not have symptoms for which they had consulted a physician before the event (in other words, were patients with no documented arrhythmic events really asymptomatic?). In contrast to these studies, Leitch et al reported no cases of sudden cardiac death in a group of 75 initially asymptomatic patients followed for a mean of 4.3 years. ${ }^{10}$ Similar conclusions about the short term outcome of asymptomatic WPW individuals have been reached by others. ${ }^{29}$ Moreover, Krahn et al showed that 19 originally healthy men who were not screened for the WPW syndrome or for asymptomatic pre-excitation and were found to have preexcitation on a routine ECG had an excellent long term prognosis. ${ }^{30}$ Munger et al also reported two sudden cardiac deaths among patients with WPW pattern over 1338 patientyears of follow up, but neither of these occurred in patients who were asymptomatic at diagnosis. ${ }^{5}$ From our results and those of previous reports, it seems that definitive treatment may be postponed until symptoms develop, as only three of 77 asymptomatic cases developed symptoms and no asymptomatic case had syncope or sudden death.

\section{STUDY LIMITATIONS}

We attempted to identify as many cases of ventricular pre-excitation as possible in the population of a defined geographical region by collecting data from a variety of health care sources within the region. However, underreporting - either because asymptomatic subjects did not have an ECG or because certain health care providers may have failed to report every case-cannot be excluded, although in the subpopulation of preoperative cases the incidence of new cases was not significantly different from that in the general population.
There was no information about the electrophysiological characteristics of the bypass tract in the majority of the symptomatic and asymptomatic patients.

Localisation of the accessory pathway on the basis of a surface 12 lead ECG rather than by electrophysiological study cannot achieve the highest accuracy. Also, multiple pathways could not be identified. Nonetheless, this study offers a rare opportunity to examine the natural history of a relatively common condition in an unselected population in whom few interventions (pharmacological or non-pharmacological) had taken place.

CONCLUSIONS

In north west Greece the WPW pattern is more common and diagnosed at a younger age in male than in female subjects. Half the patients with a WPW pattern on ECG were asymptomatic (no sex difference) at diagnosis and tended to remain so thereafter. Supraventricular tachycardia appeared at a younger age than atrial fibrillation. No sudden cardiac death occurred during a 720 patient-years follow up period. The prognosis is generally benign.

We wish to express our sincere thanks to all the doctors of the region of north west Greece for their kind contribution in collecting the data for the present study, as well as to D Iskos for his critical review of the manuscript.

1 Wellens HJJ, Brugada P, Penn OC, et al. Pre-excitation syndromes: clinical presentation, course and therapy. In: Zipes DP, Jalife J, eds. Cardiac electrophysiology: from cell to bedside. Philadelphia: WB Saunders, 1990:691-702.

2 Josephson M. Preexcitation syndromes. In: Josephson M, ed. Clinical cardiac electrophysiology. Philadelphia: Lea \& Febiger, 1993:311-416.

3 Guize L, Soria R, Chaouat JC, et al. Prevalence and course of Wolff-Parkinson-White syndrome in population of 89.

4 Wellens HJJ, Fare J, Bar FW. The Wolff-Parkinson-White syndrome. In: Mandel WJ, ed. Cardiac arrhythmias. Their mechanisms, diagnosis and management. Philadelphia: JP Lippincott, 1987:274-96.

5 Munger TM, Packer DL, Hammil SC, et al. A population Munger TM, Packer DL, Hammil SC, et al. A population
study of the natural history of Wolff-Parkinson-White syndrome in Olmsted County, Minnesota, 1953-1989. Circudrome in Olmsted County

6 Berkman NL, Lamb LE. The Wolff-Parkinson-White Berkman NL, Lamb LE. The Wolff-Parkinson-White
syndrome. A follow-up study of five to twenty-eight years. N Engl F Med. 1968;278:492-4.

7 Flensted-Jensen E. Wolff-Parkinson-White. A long-term follow-up of 47 cases. Acta Med Scand 1969;186:65-74.

8 Fitzpatrick AP, Gonzales RG, Lesh MD, et al. New algorithm for the localisation of accessory atrioventricular connections using a baseline electrocardiogram. $\mathcal{f} \mathrm{Am}$ Coll Cardiol 1994;23:107-17.

9 Rose GA, Blackburn H, Gillum RF, et al. Population studies: general aims and types. In: Cardiovascular survey methods, 2nd ed. Geneva: World Health Organisation, 1982:15-28.

10 Leitch JW, Klein GJ, Yee R, et al. Prognostic value of electrophysiology testing in asymptomatic patients with Wolff-Parkinson-White pattern. Circulation 1990;82:171823.

11 Sano S, Komori S, Amano T, et al. Prevalence of ventricular preexcitation in Japanese schoolchildren. Heart 1998;79. $374-8$.

12 Timmermans C, Smeets J, Rodriguez LM, et al. Aborted sudden death in the Wolff-Parkinson-White syndrome. Am f Cardiol 1995;76:492-4

13 Klein GJ, Prystowsky EN, Yee R, et al. Asymptomatic WolffParkinson-White: should we intervene? Circulation 1989; 80:1902-5.

14 Topaz O, Perin E, Cox M, et al. Young adult survivors of sudden cardiac arrest: analysis of invasive evaluation of 22 subjects. Am Heart f 1989;118:281-7.

15 Campbell RWF, Smith RA, Gallagher JJ, et al. Atrial fibrillation in the preexcitation syndrome. Am f Cardiol 1977;40: 514-20.

16 Bauernfeind RA, Whydham CR, Swiryn SP, et al. Paroxysmal atrial fibrillation in the Wolff-Parkinson-White syndrome. Am f Cardiol 1981;47:562-9.

17 Sharma AD, Klein GJ, Guiraudon GM, et al. Atrial fibrillation in patients with Wolff-Parkinson-White syndrome: tion in patients with Wolff-Parkinson-White syndrome:
incidence after surgical ablation of the accessory pathway. Circulation 1985;72:161-9. 
18 Robinson K, Rowland E, Krikler DM. Wolff-ParkinsonWhite syndrome: atrial fibrillation as the presenting White syndrome: atrial fibrillation as
arrhythmia. Br Heart $\mathcal{1}$ 1988;59:578-80.

19 Pietersen AH, Andersen ED, Sandoe E. Atrial fibrillation in the Wolff-Parkinson-White syndrome. Am f Cardiol 1992; 70:38-43A

20 Gallagher JJ, Pritchett ELC, Sealy WC, et al. The pre-excitation syndrome. Prog Cardiovasc Dis 1978;2:285327.

21 Schluter M, Geiger M, Siebels J, et al. Catheter ablation using radiofrequency current to cure symptomatic patients with tachyarrhythmias related to an accessory atrioventricular pathway. Circulation 1991;324:1606-11.

22 Calkins H, Langberg J, Sousa J, et al. Radiofrequency catheter ablation of accessory atrioventricular connections in 250 patients: abbreviated therapeutic approach to WolffParkinson-White. Circulation 1992;85:1337-46.

23 Swartz JF, Tracy CM, Fletcher RD. Radiofrequency endocardial catheter ablation of accessory atrioventricular pathway atrial insertion sites. Circulation 1993;87:487-99.

24 Auricchio A, Klein H, Trappe HI, et al. Lack of prognostic value of syncope in patients with Wolff-Parkinson-White syndrome. F Am Coll Cardiol 1991;12:144-50.
25 Mantakas ME, McCue CM, Miller WW. Natural history of Wolff-Parkinson-White syndrome discovered in infancy. Am f Cardiol 1978;41:1097-103.

26 Srekely P, Snaith L. Paroxysmal tachycardia in pregnancy. Br Heart f 1953;15:195-8.

27 Kounis NG, Zavras GM, Papadaki PJ, et al. Pregnancyinduced increase of supraventricular arrhythmias in Wolff-Parkinson-White syndrome. Clin Cardiol 1995;18: 137-140

28 Proudfit WL, Sterha R. Long term status and survival in Wolff-Parkinson-White syndrome. Cleve Clin 7 Med 1989; 56:601-6.

29 Beckman KJ, Gallastegui JL, Bauman JL, et al. The predictive value of electrophysiologic studies in untreated patients with Wolff-Parkinson-White syndrome. $\mathcal{F}$ Am Coll Cardiol 1990;15:640-7.

$30 \mathrm{Krahn} \mathrm{AD}$, Manfreda J, Tate RB, et al. The natural history of electrocardiographic preexcitation in men: the Manitoba follow-up study. Ann Intern Med 1992;116:456-60.

31 Klein GJ, Bashore TM, Sellers TD, et al. Ventricular fibrillation in the Wolff-Parkinson-White syndrome. $N$ Engl f Med 1979;301:1080-5.

\section{IMAGES IN CARDIOLOGY}

\section{Thrombosis of a mitral valve prosthesis}

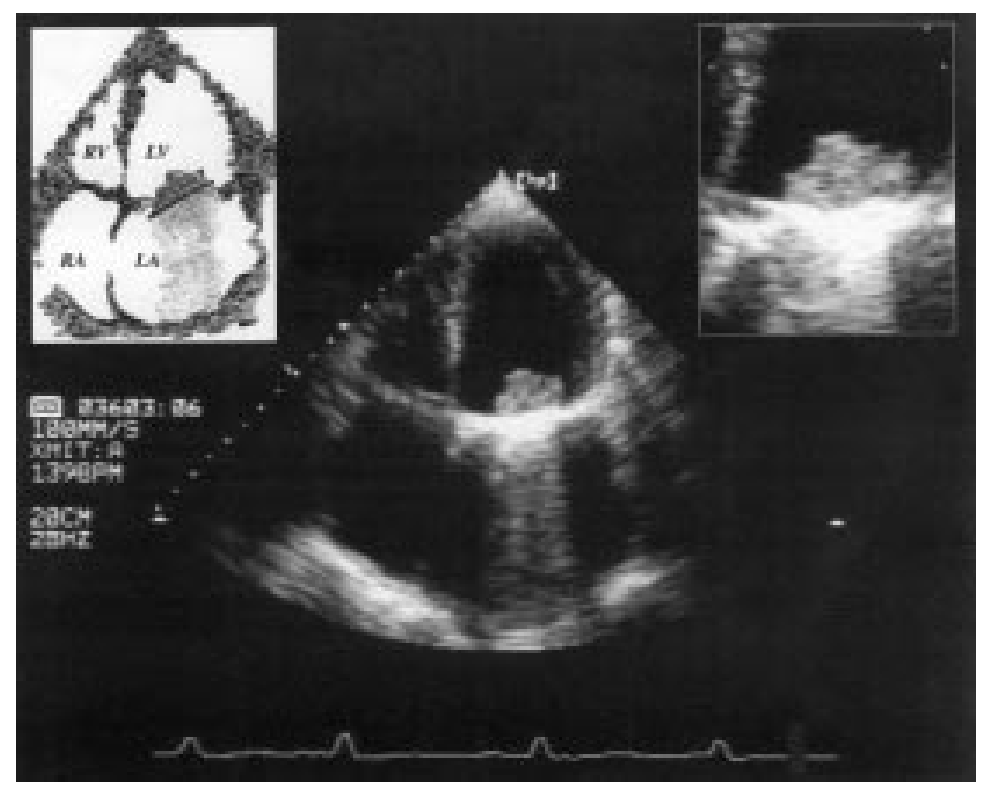

Transthoracic echocardiography of a 40 year old man showing a thrombosis of a BjörkShiley mechanical valve prosthesis in the mitral position. The patient had received Björk-Shiley prostheses in the mitral and aortic positions 25 years earlier because of rheumatic degeneration of the valves. He had chronic atrial fibrillation and a reduced left ventricular function. He presented with rapidly progressive dyspnoea and orthopnoea. He successfully underwent intravenous thrombolysis followed a few days later by a mitral valve prosthesis replacement. (Top left, artistic representation of the thrombus on the prosthesis. Note the acoustic shadow produced by the prosthesis in the left atrium. Top right, magnification of the thrombosed prosthesis. LA, left atrium; LV, left ventricle; RA, right atrium; LA, left ventricle.) 